\title{
Selective Methanol Carbonylation to Acetic Acid on Heterogeneous Atomically Dispersed $\mathrm{ReO}_{4} / \mathrm{SiO}_{2}$ Catalysts
}

Ji Qi ${ }^{1}$, Jordan Finzel ${ }^{1}$, Hossein Robatjazi ${ }^{1}$, Mingjie Xu ${ }^{2,3,4}$, Adam S. Hoffman $^{5}$, Simon R. Bare ${ }^{5}$, Xiaoqing Pan ${ }^{2,3,4}$, Phillip Christopher ${ }^{1 *}$

1. Department of Chemical Engineering, University of California, Santa Barbara, Santa Barbara, California 93117, United States.

2. Department of Materials Science and Engineering, 3. Department of Physics and Astronomy, 4. Irvine Materials Research Institute (IMRI), University of California Irvine, Irvine, California 92697, United States.

5. Stanford Synchrotron Radiation Light Source, SLAC National Accelerator Laboratory, Menlo Park, California 94025, United States

*Corresponding Author Email: pchristopher@ucsb.edu

Figure S1: EXAFS spectra and fit for Re metal.

Figure S2: Calibration curve for Re oxidation state vs. XANES edge energy.

Figure S3: XPS analysis for Re oxidation state.

Figure S4: EXAFS spectra in k-space and R-space for oxidized samples.

Figure S5: Scattering paths for $\mathrm{Re}_{2} \mathrm{O}_{7}$.

Figure S6: Acetic acid production rate as a function of superficial velocity.

Figure S7: Methyl acetate and formaldehyde production rate as the function of temperature.

Figure S8: $\mathrm{NH}_{3}-\mathrm{TPD}$

Figure S9: Comparison of DME and methanol carbonylation rate

Figure S10: Comparison of in situ FTIR spectra for $\mathrm{SiO}_{2}$ and $5 \mathrm{wt} \% \mathrm{Re} / \mathrm{SiO}_{2}$.

Figure S11: Comparison of short and long Re-O scattering paths.

Figure S12: Dimethyl Ether (DME) formation rate comparison on $10 \mathrm{wt} . \% \mathrm{Re} / \mathrm{SiO}_{2}$ and 0.2 wt. $\% \mathrm{Rh} / 10$ wt. $\% \mathrm{Re}-\mathrm{SiO}_{2}$.

Figure S13: Arrhenius plots showing the acetic acid (AA) formation rate on $10 \mathrm{wt} . \% \mathrm{Re} / \mathrm{SiO}_{2}$ and $0.2 \mathrm{wt} . \% \mathrm{Rh} / 10 \mathrm{wt} . \% \mathrm{Re}-\mathrm{SiO}_{2}$.

Table S1: Mass balance for $1 \mathrm{wt} . \% \mathrm{Re} / \mathrm{SiO}_{2}$.

Table S2: Mass balance for $20 \mathrm{wt} . \% \mathrm{Re} / \mathrm{SiO}_{2}$.

Table S3: Mass balance for high conversion on $10 \mathrm{wt} . \% \mathrm{Re} / \mathrm{SiO}_{2}$.

Table S4: The amount of catalyst used for methanol carbonylation reactivity measurements.

Table S5: The amount of catalysts used to promote methanol conversion.

Table S6: Structural parameters for EXAFS fits.

Calculation of methanol conversion to each product

Rate Expression Derivation 


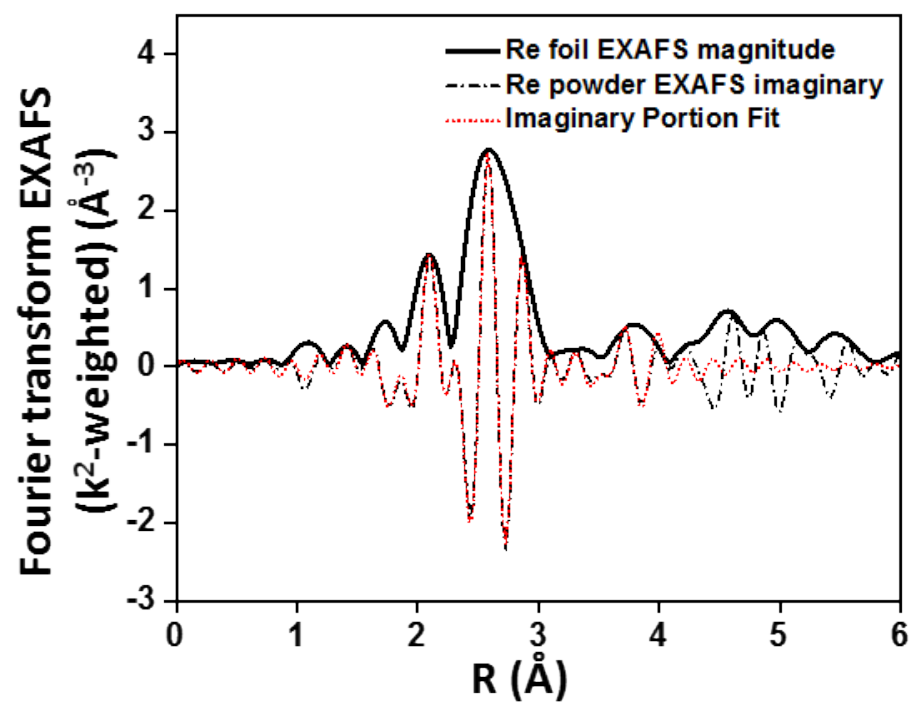

Figure S1. Fitting for the Re $\mathrm{L}_{3}$ edge of Re metal powder used to determine $\mathrm{S}_{0}{ }^{2}$ for EXAFS fitting of Re/SiO${ }_{2} \mathrm{samples}_{\text {. }}$ Fits were performed over a $\mathrm{k}$ range of $3-14 \AA^{-1}$ and an $\mathrm{R}$ range of 1.2-2.8 $\AA$.
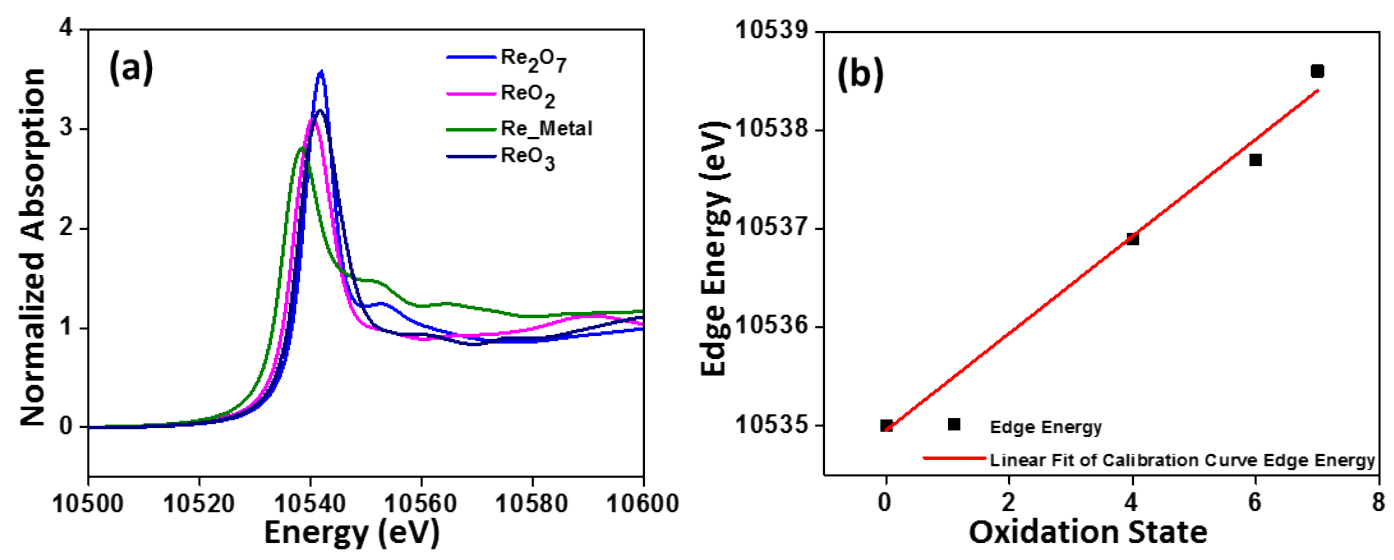

Figure S2. (a) XANES spectra for Re reference compounds with different formal oxidation states. (b) Calibration curve for relationship between oxidation state and $\mathrm{Re}_{2}$-edge energy, defined as the maximum of the first derivative of the XANES spectrum. The Re $\mathrm{L}_{3}$ edge energy for metallic Re was set to $10535.0 \mathrm{eV}$. 


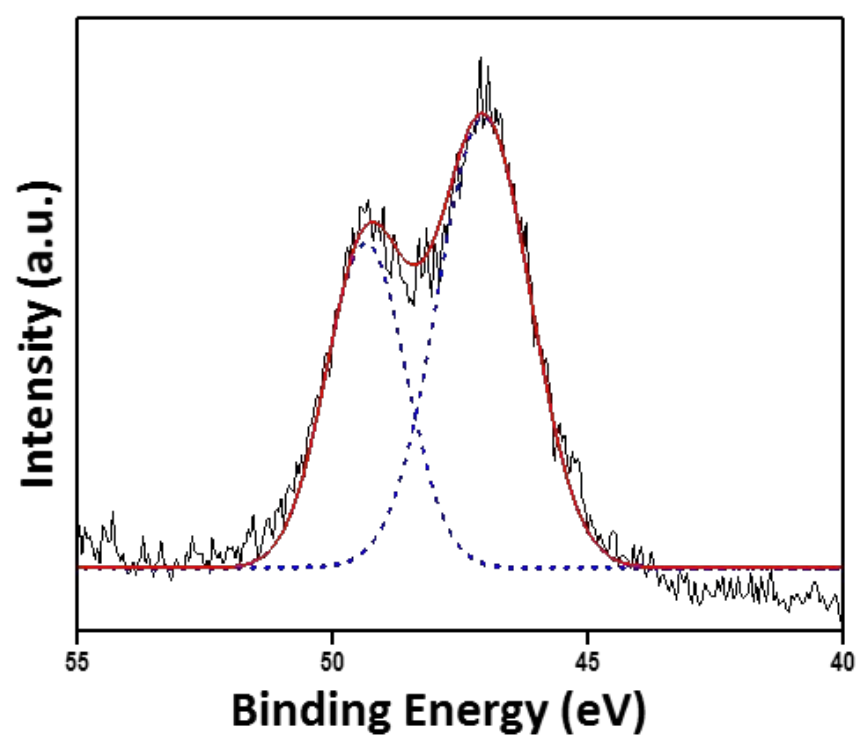

Figure S3. X-ray photoelectron spectrum (XPS) of the $5 \mathrm{wt} . \% \mathrm{Re} / \mathrm{SiO}_{2}$ following oxidation at $350{ }^{\circ} \mathrm{C}$ for $1 \mathrm{~h}$. The sample was isolated from atmosphere and was transferred to the XPS chamber using an air-tight specimen holder with sample preparation in a glove box. Two peaks at 46.9 and $49.2 \mathrm{eV}$ correspond to $\operatorname{Re} 4 \mathrm{f}_{7 / 2}$ and $\operatorname{Re}_{5 / 2}$ of $\operatorname{Re}^{+7}$. ${ }^{1,2}$
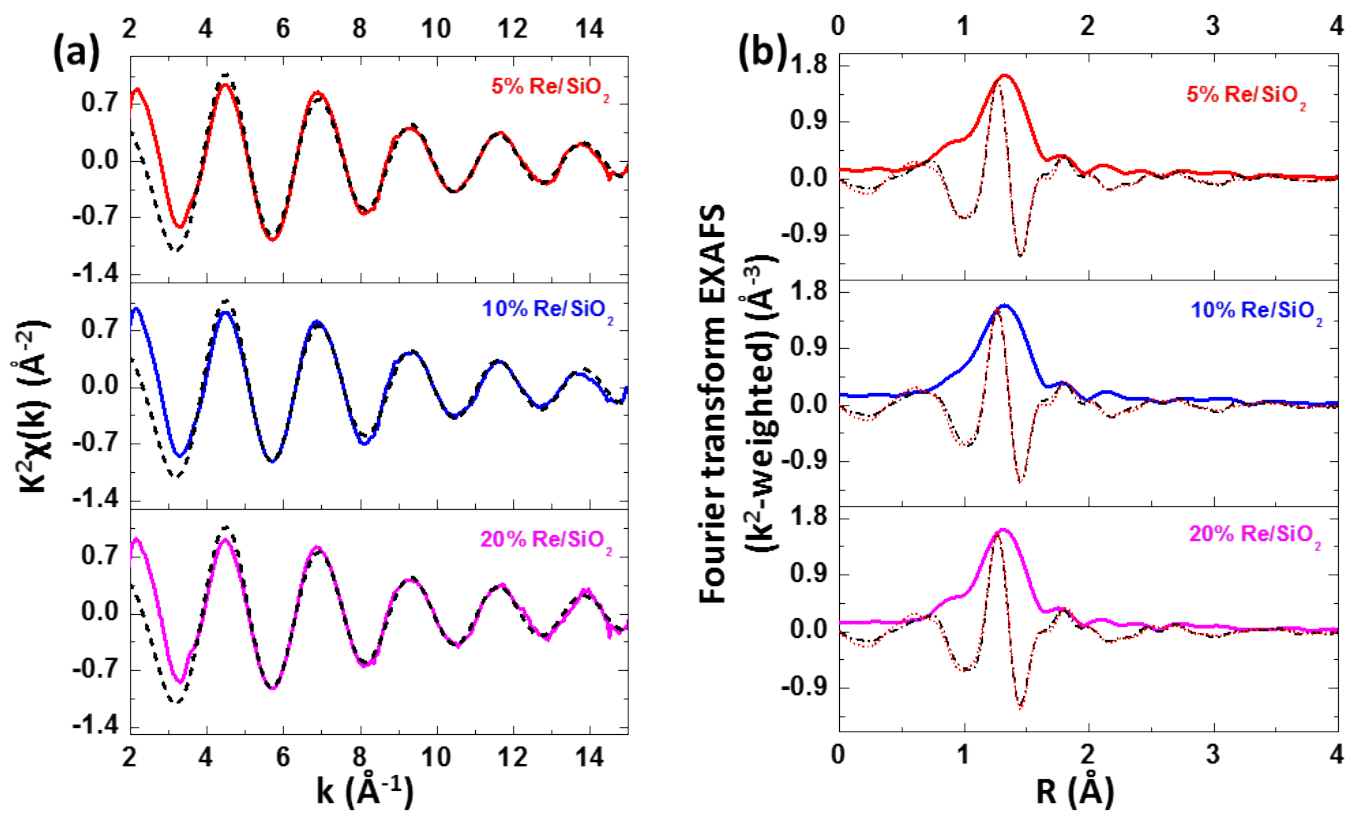

Figure S4. (a) EXAFS spectra in k-space of various loadings of $\mathrm{Re} / \mathrm{SiO}_{2}$ after oxidation at $350{ }^{\circ} \mathrm{C}$ for $1 \mathrm{~h}$. The fit is shown as dotted lines and was performed using a Hanning window over a k range 3.9-14 $\AA^{-1}$. (b) The FT EXAFS spectra of the spectra in Figure S4(a) with fits performed over an R range 1.0-2.2 $\AA$. Black dashed lines show the imaginary portion of the FT while red dashed lines show the fit to the imaginary portion. 


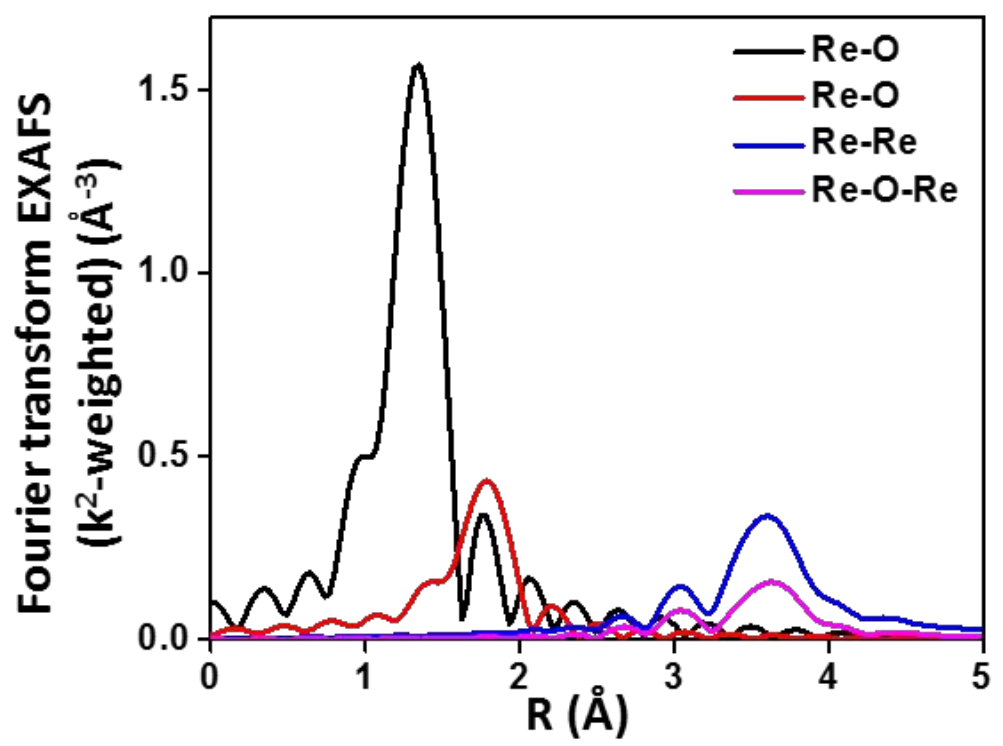

Figure S5. Magnitude of the FT of the calculated single scattering paths for $\mathrm{Re}_{2} \mathrm{O}_{7}$ (mp-1016092) showing relative position and amplitude of short-R range Re-O components vs. longer R range features associated with oligomeric Re.

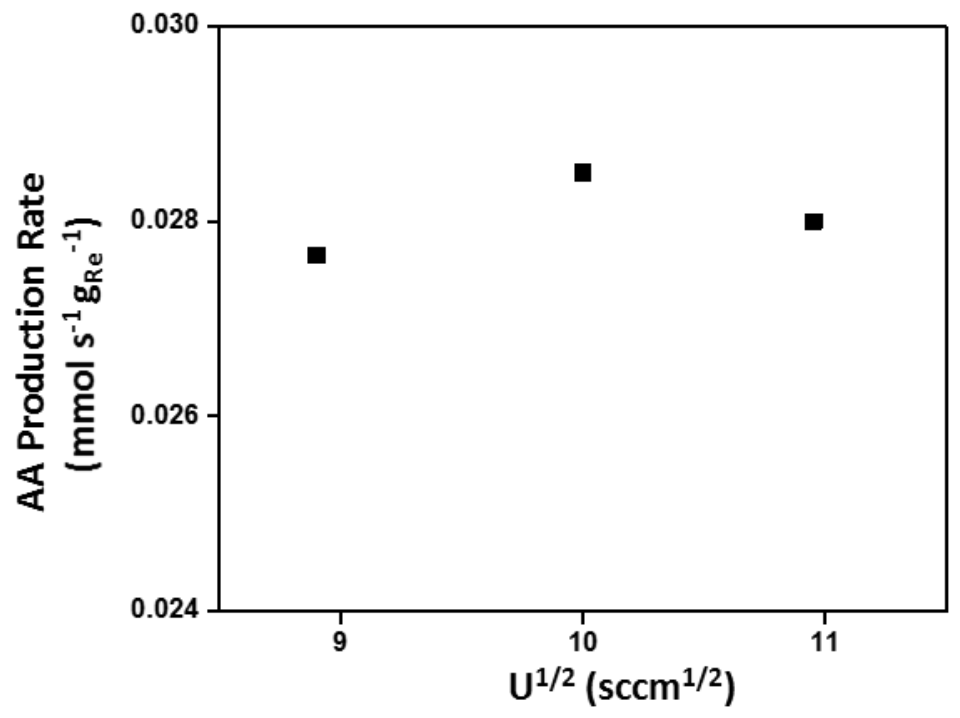

Figure S6. AA production rate for $10 \mathrm{wt} . \% \mathrm{Re} / \mathrm{SiO}_{2}$ measured at $280{ }^{\circ} \mathrm{C}$ and $30 \mathrm{mbar}$ methanol and $\mathrm{CO}$ (balance $\mathrm{He}$ ) plotted as a function of the square root of the superficial velocity. The rates were observed to be independent of the superficial velocity suggesting a lack of mass transfer limitations. A superficial velocity of $100 \mathrm{~cm}^{3} \mathrm{~min}^{-1}\left(20 \mathrm{~cm}^{3}\right.$ $\mathrm{min}^{-1}$ of He to purge methanol and $30 \mathrm{~cm}^{3} \mathrm{~min}^{-1}$ of $10 \% \mathrm{CO} / \mathrm{He}$ with $50 \mathrm{~cm}^{3} \mathrm{~min}^{-1}$ of $\mathrm{He}$ ) was chosen for all kinetic experiments. 

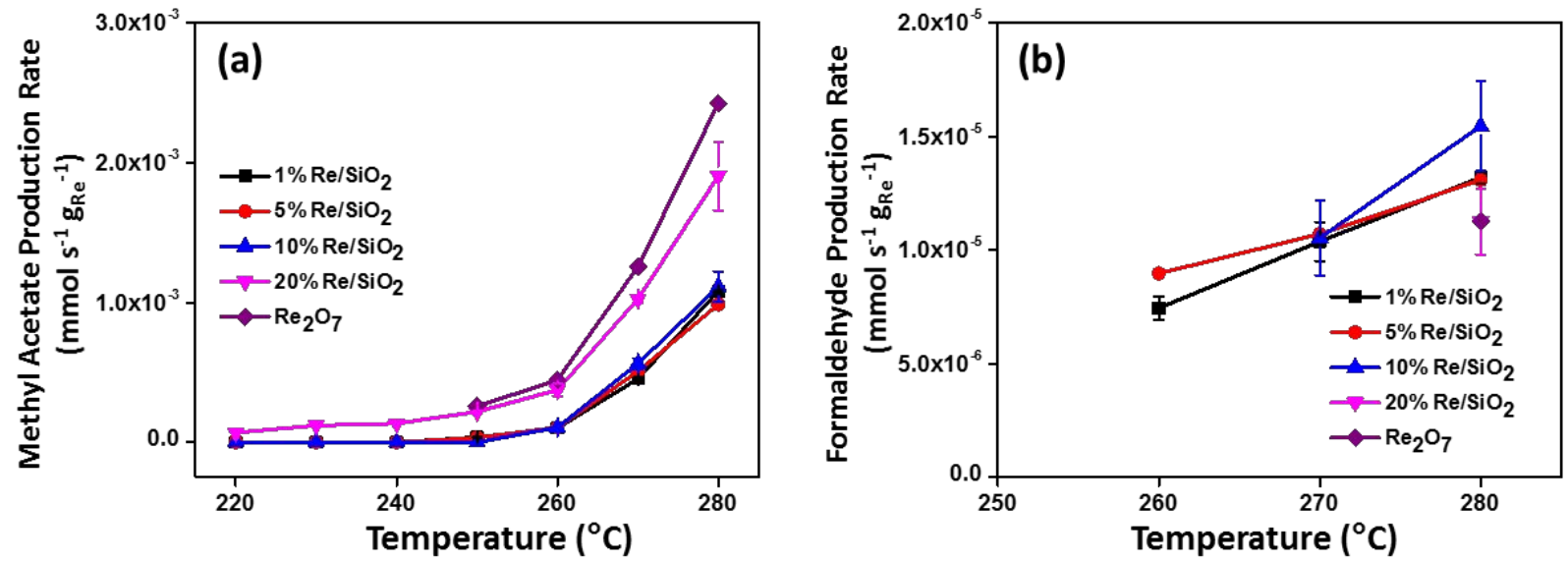

Figure S7. Production rate of methyl acetate (left) and formaldehyde (right) measured at 30 mbar methanol and 30 mbar $\mathrm{CO}$ (balance $\mathrm{He}$ ) as a function of temperature on $1,5,10$ and $20 \mathrm{wt} . \% \mathrm{Re} / \mathrm{SiO}_{2}$. There is no measurable formaldehyde formation at lower temperature.

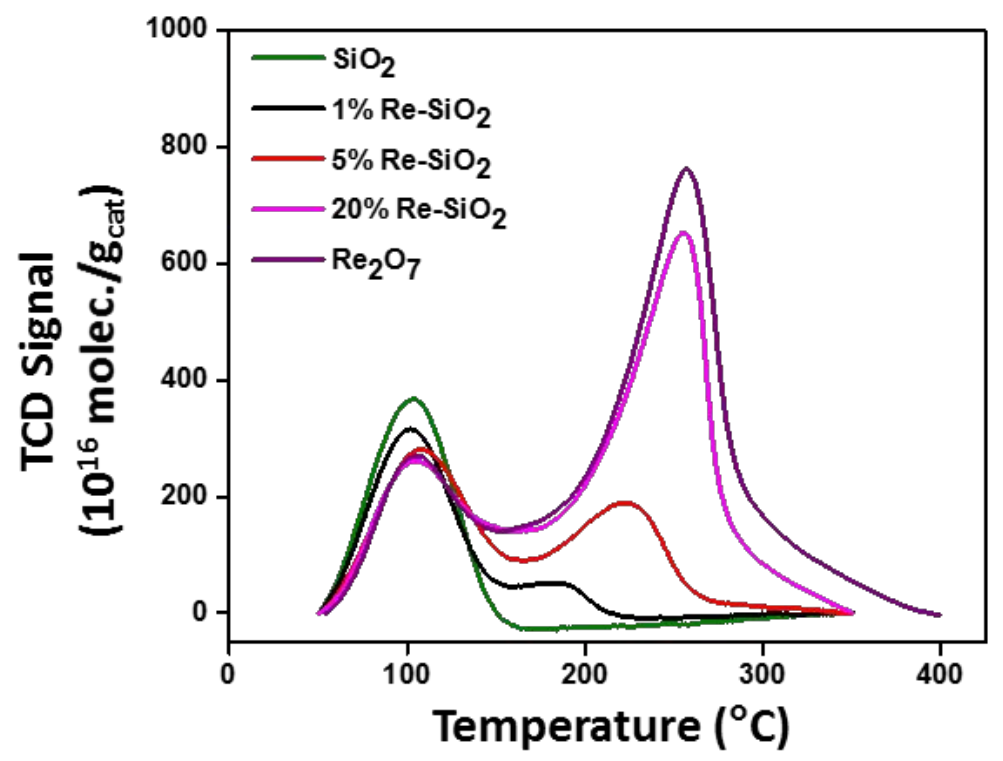

Figure S8. $\mathrm{NH}_{3}$-TPD for $\mathrm{SiO}_{2}, 1$ wt. $\% \mathrm{Re} / \mathrm{SiO}_{2}, 5$ wt.\%Re/ $/ \mathrm{SiO}_{2}, 20$ wt. $\% \mathrm{Re} / \mathrm{SiO}_{2}$ and bulk $\mathrm{Re}_{2} \mathrm{O}_{7}($ Sigma-Aldrich, 1314-68-7) physically mixed with $\mathrm{SiO}_{2}$ (the amount of $\mathrm{Re}_{2} \mathrm{O}_{7}$ and $\mathrm{SiO}_{2}$ were adjusted to provide 20 wt.\%Re/ $/ \mathrm{SiO}_{2}$ ). All catalysts were pretreated in $\mathrm{O}_{2}$ at $350{ }^{\circ} \mathrm{C}$ for $1 \mathrm{~h}$. A mixture of $10 \% \mathrm{NH}_{3} / 90 \% \mathrm{He}$ was flown over the catalyst at $50{ }^{\circ} \mathrm{C}$ for $1 \mathrm{~h}$. The TPD measurements were carried out in the range $50-350{ }^{\circ} \mathrm{C}$ at a heating rate of $10{ }^{\circ} \mathrm{C} / \mathrm{min}$ under $\mathrm{He}$. The peak at $100{ }^{\circ} \mathrm{C}$ represents the weak acid sites on $\mathrm{SiO}_{2}$ and the $200-250{ }^{\circ} \mathrm{C}$ peak represents the acid sites created by $\mathrm{ReO}_{\mathrm{x}}$ species. 


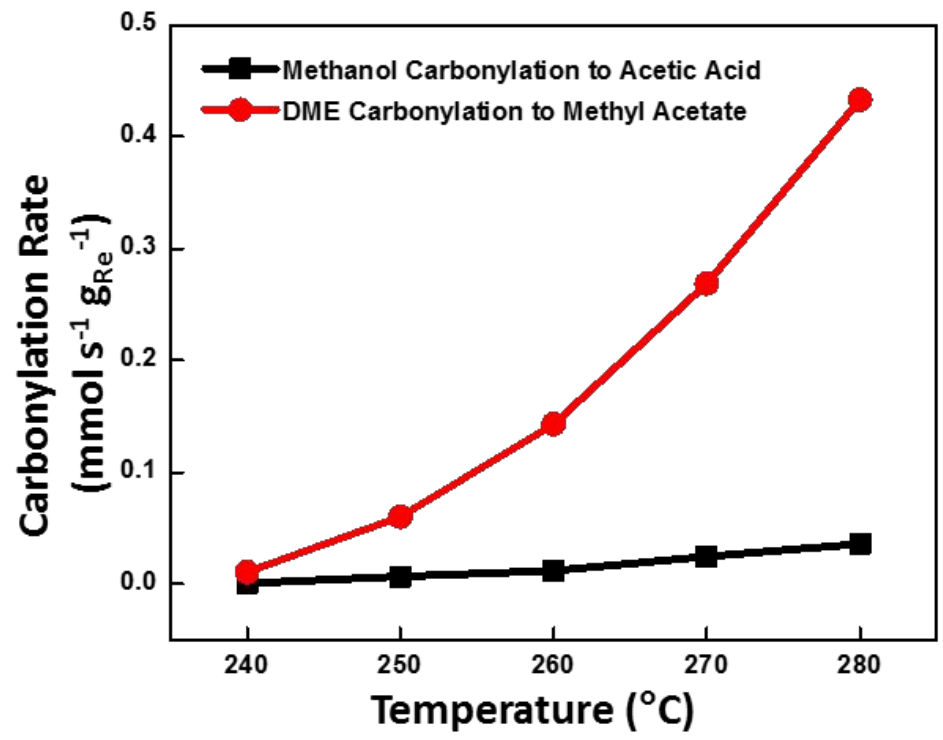

Figure S9. Reaction rate for methanol carbonylation to acetic acid and dimethyl ether carbonylation to methyl acetate on $5 \mathrm{wt} . \% \mathrm{Re} / \mathrm{SiO}_{2}$ from 240 to $280^{\circ} \mathrm{C}$ under $30 \mathrm{mbar}$ methanol or dimethyl ether and $30 \mathrm{mbar} \mathrm{CO}$ (balance $\mathrm{He})$.

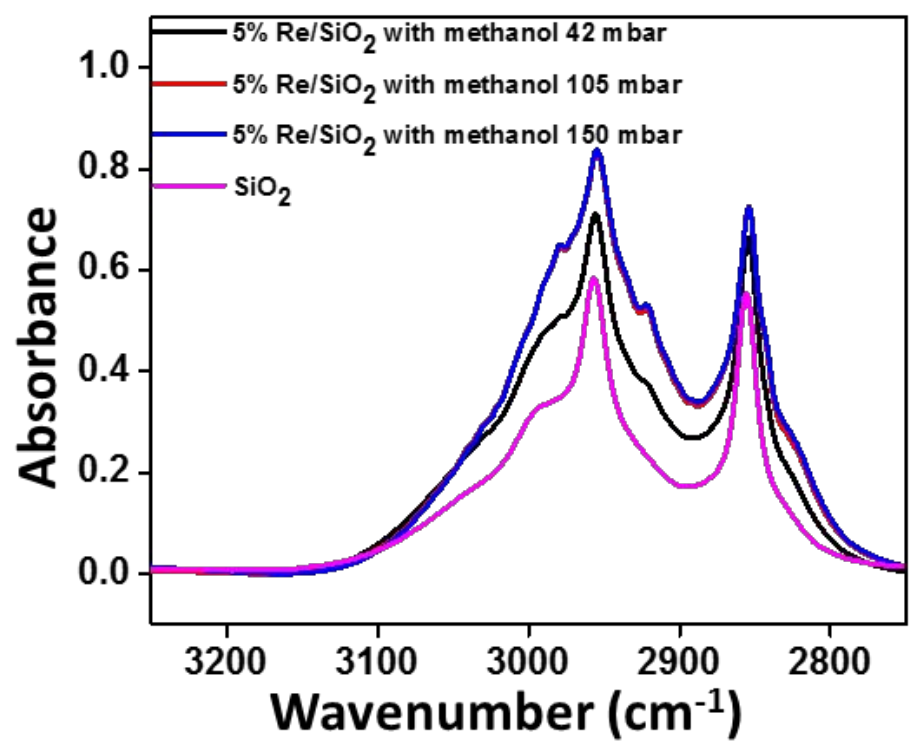

Figure S10. Comparison of in situ FTIR spectra for $\mathrm{SiO}_{2}$ and 5 wt.\% Re/SiO 2 . The 5 wt.\% Re/SiO $\mathrm{Sample}_{2}$ was oxidized at $350{ }^{\circ} \mathrm{C}$ for $1 \mathrm{~h}$ before being exposed to 42,105 and 150 mbar methanol with CO partial pressure the same as 30 mbar (balanced by $\mathrm{He}$ ). The $\mathrm{SiO}_{2}$ sample was oxidized at $350{ }^{\circ} \mathrm{C}$ for $1 \mathrm{~h}$ before being exposed to $150 \mathrm{mbar}$ methanol with $\mathrm{CO}$ partial pressure as $30 \mathrm{mbar}$ (balanced by He). The spectra were collected under methanol and CO. On $\mathrm{SiO}_{2}$, two strong bands at 2954 and $2854 \mathrm{~cm}^{-1}$ correspond to intact methanol on $\mathrm{SiO}_{2}$ and a weak shoulder at 2996 $\mathrm{cm}^{-1}$ represents $\mathrm{CH}_{3} \mathrm{O}$ - on $\mathrm{SiO}_{2}{ }^{3,4}$ 


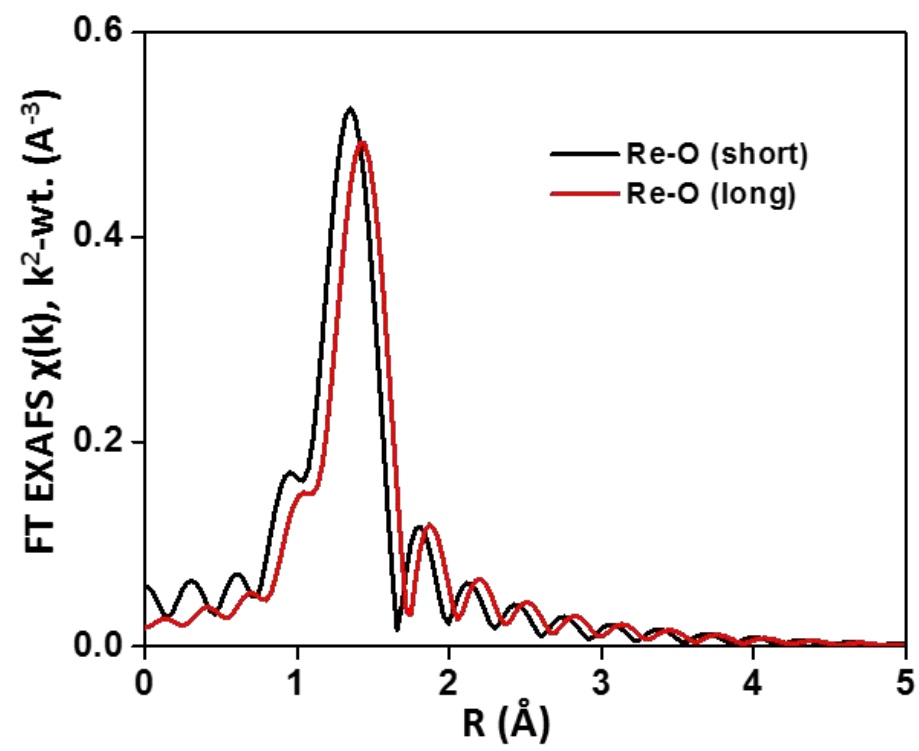

Figure S11. Magnitude of FT of the short and long Re-O scattering paths used in this work. For both paths, $\mathrm{S}_{\mathrm{o}}{ }^{2}=0.73$, $\mathrm{N}=1$, and $\sigma^{2}=0$ in order to compare the relative magnitude of the two paths. There is a decreased peak intensity for longer $\mathrm{Re}-\mathrm{O}$ (single) bonds compared to the shorter $\mathrm{Re}=\mathrm{O}$ (double) bond. The decrease in intensity for the major peak for in-situ vs oxidized data (Figure 6c) may be consistent with the proposed mechanism in which Re-O single bond coordination increases and $\mathrm{Re}=\mathrm{O}$ double bond coordination decreases.

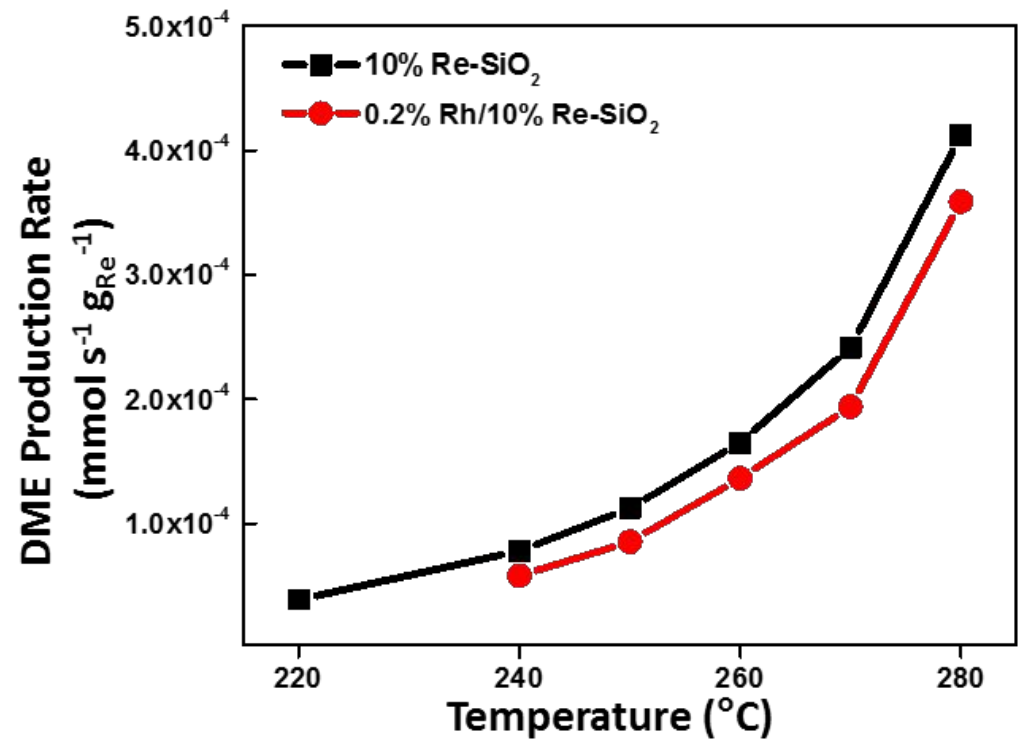

Figure S12. Dimethyl Ether (DME) formation rate on $10 \mathrm{wt} . \% \mathrm{Re} / \mathrm{SiO}_{2}$ and $0.2 \mathrm{wt} . \% \mathrm{Rh} / 10 \mathrm{wt} . \% \mathrm{Re}-\mathrm{SiO} \mathrm{during}$ methanol carbonylation from 200 to $280^{\circ} \mathrm{C}$ under 30 mbar methanol and $\mathrm{CO}$ (balance $\mathrm{He}$ ). 


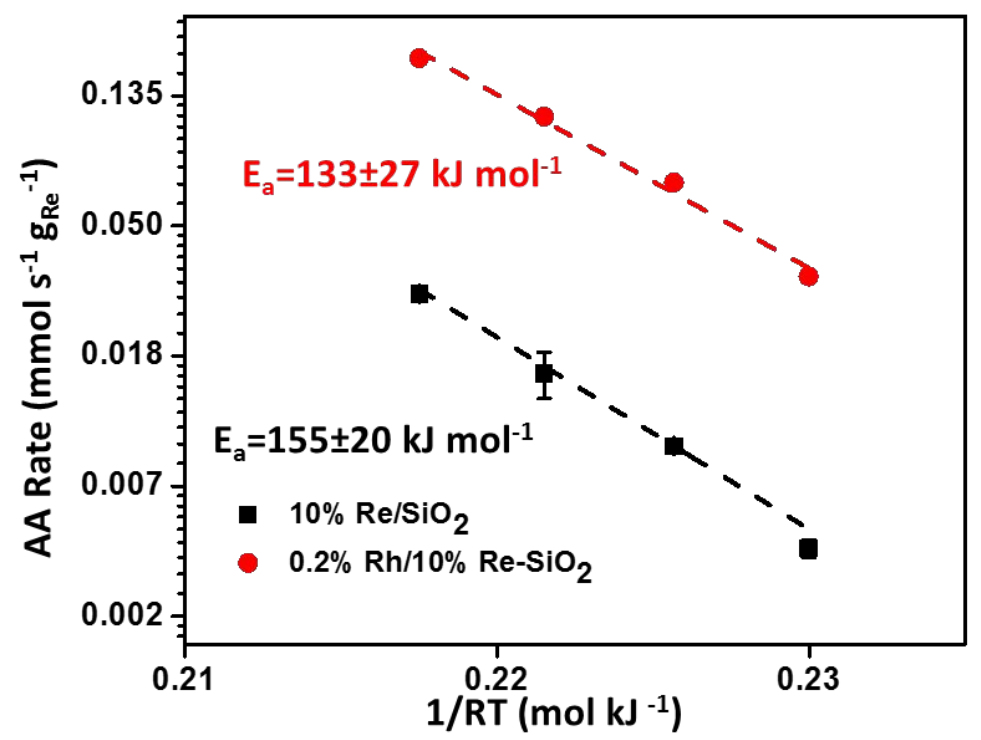

Figure S13. Arrhenius plots of the acetic acid (AA) formation rate on 10 wt. $\%$ Re/SiO 2 and 0.2 wt. $\%$ Rh/10 wt.\%Re$\mathrm{SiO}_{2}$ during methanol carbonylation from 250 to $280^{\circ} \mathrm{C}$ under $30 \mathrm{mbar}$ methanol and $\mathrm{CO}$ (balance He). The error bars on $10 \mathrm{wt} . \% \mathrm{Re} / \mathrm{SiO}_{2}$ represent the standard deviation calculated from 2 independently run reactions. The error on Eapp estimates is the $90 \%$ confidence interval of the fit. 
Table S1: Mass balance for methanol carbonylation on $1 \mathrm{wt} . \% \mathrm{Re} / \mathrm{SiO}_{2}$ from 220 to $280{ }^{\circ} \mathrm{C}$ at $30 \mathrm{mbar}$ methanol and $\mathrm{CO}$ (balance $\mathrm{He}$ ).

a: methanol conversion to each product; $b$ : total methanol conversion from the summation of products; c: methanol conversion based on methanol loss. The analysis method is noted for each calculation.

\begin{tabular}{|c|c|c|c|c|c|c|c|c|}
\hline & \multicolumn{2}{|c|}{$\begin{array}{c}\text { Conversion to } \\
\text { AA }^{\mathrm{a}}\end{array}$} & \multicolumn{2}{|c|}{$\begin{array}{c}\text { Conversion to } \\
\text { DME }^{\mathrm{a}}\end{array}$} & \multicolumn{2}{|c|}{$\begin{array}{c}\text { Conversion to } \\
\text { MA }^{\mathrm{a}}\end{array}$} & \multicolumn{2}{|c|}{$\begin{array}{l}\text { Conversion to } \\
\text { Formaldehyde }^{\text {a }}\end{array}$} \\
\hline & MS & $\mathrm{GC}$ & MS & $\mathrm{GC}$ & MS & $\mathrm{GC}$ & MS & $\mathrm{GC}$ \\
\hline $220^{\circ} \mathrm{C}$ & $7.2 \mathrm{E}-05$ & & $5.8 \mathrm{E}-05$ & & & & & \\
\hline $230^{\circ} \mathrm{C}$ & $4.5 \mathrm{E}-04$ & & $7.4 \mathrm{E}-05$ & & & & & \\
\hline $240^{\circ} \mathrm{C}$ & $8.8 \mathrm{E}-04$ & 3.7E-03 & $1.1 \mathrm{E}-04$ & $1.1 \mathrm{E}-04$ & & & & \\
\hline $250^{\circ} \mathrm{C}$ & $6.9 \mathrm{E}-03$ & $6.5 \mathrm{E}-03$ & $1.6 \mathrm{E}-04$ & $1.5 \mathrm{E}-04$ & 3.4E-05 & $3.2 \mathrm{E}-05$ & & \\
\hline $260^{\circ} \mathrm{C}$ & $1.3 \mathrm{E}-02$ & $1.2 \mathrm{E}-02$ & $1.9 \mathrm{E}-04$ & $1.9 \mathrm{E}-04$ & 9.3E-05 & $9.1 \mathrm{E}-05$ & $7.0 \mathrm{E}-06$ & $6.4 \mathrm{E}-06$ \\
\hline $270{ }^{\circ} \mathrm{C}$ & $2.3 \mathrm{E}-02$ & $2.4 \mathrm{E}-02$ & $3.0 \mathrm{E}-04$ & $3.1 \mathrm{E}-04$ & 4.6E-04 & 4.0E-04 & $8.8 \mathrm{E}-06$ & $9.8 \mathrm{E}-06$ \\
\hline \multirow[t]{3}{*}{$280^{\circ} \mathrm{C}$} & $3.5 \mathrm{E}-02$ & 3.6E-02 & $4.6 \mathrm{E}-04$ & 4.7E-04 & 9.7E-04 & $9.8 \mathrm{E}-04$ & $1.0 \mathrm{E}-05$ & $1.4 \mathrm{E}-05$ \\
\hline & \multicolumn{3}{|c|}{$\begin{array}{l}\text { Methanol Conversion } \\
\text { (product sum) }^{\mathrm{b}}\end{array}$} & \multicolumn{2}{|c|}{$\begin{array}{l}\text { Methanol Conversion } \\
\text { (reactant loss) }^{c}\end{array}$} & \multicolumn{3}{|c|}{$\begin{array}{l}\text { Mass Balance Closure } \\
\qquad(100 \%)\end{array}$} \\
\hline & MS & & & MS & $\mathrm{GC}$ & $M$ & & $\mathrm{GC}$ \\
\hline $220^{\circ} \mathrm{C}$ & $1.3 \mathrm{E}-04$ & & & $1.5 \mathrm{E}-04$ & & 90 & & \\
\hline $230^{\circ} \mathrm{C}$ & $5.3 \mathrm{E}-04$ & & & $5.7 \mathrm{E}-04$ & & 92 & & \\
\hline $240{ }^{\circ} \mathrm{C}$ & $9.9 \mathrm{E}-04$ & & & $1.1 \mathrm{E}-04$ & $4.1 \mathrm{E}-03$ & 92 & & 92 \\
\hline $250^{\circ} \mathrm{C}$ & $7.1 \mathrm{E}-03$ & & & $7.7 E-03$ & $7.0 \mathrm{E}-03$ & 92 & & 92 \\
\hline $260^{\circ} \mathrm{C}$ & $1.3 \mathrm{E}-02$ & & & $1.4 \mathrm{E}-02$ & $1.3 \mathrm{E}-02$ & 95 & & 95 \\
\hline $270{ }^{\circ} \mathrm{C}$ & $2.4 \mathrm{E}-02$ & & & $2.5 \mathrm{E}-02$ & $2.6 \mathrm{E}-02$ & 95 & & 95 \\
\hline $280^{\circ} \mathrm{C}$ & $3.6 \mathrm{E}-02$ & & & $3.8 \mathrm{E}-02$ & $3.9 \mathrm{E}-02$ & 96 & & 96 \\
\hline
\end{tabular}


Table S2: Mass balance for methanol carbonylation on $20 \mathrm{wt} . \% \mathrm{Re} / \mathrm{SiO}_{2}$ from 220 to $280{ }^{\circ} \mathrm{C}$ at $30 \mathrm{mbar}$ methanol and $\mathrm{CO}$ (balance $\mathrm{He}$ ).

a: methanol conversion to each product; $b$ : total methanol conversion from the summation of products; c: methanol conversion based on methanol loss. The analysis method is noted for each calculation.

\begin{tabular}{|c|c|c|c|c|c|c|c|c|}
\hline & \multicolumn{2}{|c|}{$\begin{array}{c}\text { Conversion to } \\
\text { AA }^{a}\end{array}$} & \multicolumn{2}{|c|}{$\begin{array}{c}\text { Conversion to } \\
\text { DME }^{a}\end{array}$} & \multicolumn{2}{|c|}{$\begin{array}{c}\text { Conversion to } \\
\text { MA }^{a}\end{array}$} & \multicolumn{2}{|c|}{$\begin{array}{l}\text { Conversion to } \\
\text { Formaldehyde }\end{array}$} \\
\hline & MS & GC & MS & GC & MS & GC & MS & GC \\
\hline $220^{\circ} \mathrm{C}$ & $1.3 \mathrm{E}-04$ & & $1.8 \mathrm{E}-05$ & & $6.2 \mathrm{E}-05$ & $5.6 \mathrm{E}-05$ & & \\
\hline $230^{\circ} \mathrm{C}$ & $1.2 \mathrm{E}-04$ & & $7.3 \mathrm{E}-05$ & & $1.1 \mathrm{E}-04$ & $1.2 \mathrm{E}-04$ & & \\
\hline $240^{\circ} \mathrm{C}$ & $4.5 \mathrm{E}-05$ & & $1.9 \mathrm{E}-04$ & & $1.2 \mathrm{E}-04$ & $1.4 \mathrm{E}-04$ & & \\
\hline $250^{\circ} \mathrm{C}$ & $2.7 \mathrm{E}-04$ & $1.8 \mathrm{E}-04$ & $7.6 \mathrm{E}-04$ & $9.4 \mathrm{E}-04$ & $2.0 \mathrm{E}-04$ & $1.9 \mathrm{E}-04$ & & \\
\hline $260^{\circ} \mathrm{C}$ & $7.0 \mathrm{E}-04$ & $8.8 \mathrm{E}-04$ & $3.0 \mathrm{E}-03$ & $2.5 \mathrm{E}-03$ & $3.1 E-04$ & $3.6 \mathrm{E}-04$ & & \\
\hline $270^{\circ} \mathrm{C}$ & $3.2 \mathrm{E}-03$ & $2.3 \mathrm{E}-03$ & $8.2 \mathrm{E}-03$ & $7.3 \mathrm{E}-03$ & $9.4 \mathrm{E}-04$ & $9.0 \mathrm{E}-04$ & & \\
\hline \multirow[t]{3}{*}{$280^{\circ} \mathrm{C}$} & $6.2 \mathrm{E}-03$ & $4.4 \mathrm{E}-03$ & $1.8 \mathrm{E}-02$ & $2.4 \mathrm{E}-02$ & $1.6 \mathrm{E}-03$ & $1.9 \mathrm{E}-03$ & $9.2 \mathrm{E}-06$ & $1.1 \mathrm{E}-05$ \\
\hline & \multicolumn{3}{|c|}{$\begin{array}{l}\text { Methanol Conversion } \\
\text { (product sum) }^{\mathrm{b}}\end{array}$} & \multicolumn{2}{|c|}{$\begin{array}{l}\text { Methanol Conversion } \\
\text { (reactant loss) }^{c}\end{array}$} & \multicolumn{3}{|c|}{$\begin{array}{c}\text { Mass Balance Closure } \\
\qquad(100 \%)\end{array}$} \\
\hline & MS & & & MS & GC & MS & & GC \\
\hline $220^{\circ} \mathrm{C}$ & $2.1 E-04$ & & & $2.3 \mathrm{E}-04$ & $6.7 \mathrm{E}-05$ & 92 & & 92 \\
\hline $230^{\circ} \mathrm{C}$ & $3.0 \mathrm{E}-04$ & & & $3.2 \mathrm{E}-04$ & $1.3 \mathrm{E}-04$ & 92 & & 93 \\
\hline $240^{\circ} \mathrm{C}$ & $3.6 \mathrm{E}-04$ & & & $3.9 \mathrm{E}-04$ & $1.5 \mathrm{E}-04$ & 93 & & 93 \\
\hline $250^{\circ} \mathrm{C}$ & $1.2 \mathrm{E}-03$ & & & $1.3 \mathrm{E}-03$ & $1.4 \mathrm{E}-03$ & 94 & & 94 \\
\hline $260^{\circ} \mathrm{C}$ & $4.0 \mathrm{E}-03$ & & & $4.2 \mathrm{E}-03$ & $3.9 \mathrm{E}-03$ & 95 & & 95 \\
\hline $270^{\circ} \mathrm{C}$ & $1.2 \mathrm{E}-02$ & & & $1.3 \mathrm{E}-02$ & $1.1 \mathrm{E}-02$ & 96 & & 96 \\
\hline $280^{\circ} \mathrm{C}$ & $2.6 \mathrm{E}-02$ & & & $2.7 E-02$ & $3.2 \mathrm{E}-02$ & 96 & & 95 \\
\hline
\end{tabular}


Table S3: Mass balance for methanol carbonylation on $10 \mathrm{wt} . \% \mathrm{Re} / \mathrm{SiO}_{2}$ with $600 \mathrm{mg}$ catalyst loading 220 to $280{ }^{\circ} \mathrm{C}$ at 30 mbar methanol and $30 \mathrm{mbar} \mathrm{CO}$ (balance $\mathrm{He}$ ).

a: methanol conversion to each product; $b$ : methanol conversion from the summation of products; $c$ : methanol conversion based on methanol signal. All analysis was by mass spectrometry.

\begin{tabular}{|c|c|c|c|c|c|c|}
\hline & Conversion to $\mathrm{AA}^{\mathrm{a}}$ & \multicolumn{2}{|c|}{ Conversion to $\mathrm{DME}^{\mathrm{a}}$} & \multicolumn{2}{|c|}{ Conversion to $\mathrm{MA}^{\mathrm{a}}$} & $\begin{array}{l}\text { Conversion to } \\
\text { Formaldehyde }^{a}\end{array}$ \\
\hline $240^{\circ} \mathrm{C}$ & $9.6 \mathrm{E}-02$ & & $2.0 \mathrm{E}-03$ & \multicolumn{2}{|c|}{$1.3 \mathrm{E}-03$} & \\
\hline $250^{\circ} \mathrm{C}$ & $1.8 \mathrm{E}-01$ & & $3.0 \mathrm{E}-03$ & \multicolumn{2}{|c|}{$2.0 \mathrm{E}-03$} & \\
\hline $260^{\circ} \mathrm{C}$ & $2.8 \mathrm{E}-01$ & & $4.3 \mathrm{E}-03$ & \multicolumn{2}{|c|}{$2.0 \mathrm{E}-03$} & $1.4 \mathrm{E}-04$ \\
\hline $270^{\circ} \mathrm{C}$ & $3.7 \mathrm{E}-01$ & & $7.4 \mathrm{E}-03$ & \multicolumn{2}{|c|}{$9.6 \mathrm{E}-03$} & $2.1 \mathrm{E}-04$ \\
\hline $280^{\circ} \mathrm{C}$ & $5.0 \mathrm{E}-01$ & & $1.1 \mathrm{E}-02$ & \multicolumn{2}{|c|}{$1.9 \mathrm{E}-02$} & $2.5 \mathrm{E}-04$ \\
\hline & \multicolumn{2}{|c|}{ 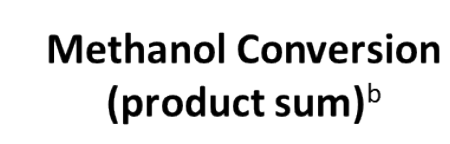 } & \multicolumn{2}{|c|}{$\begin{array}{l}\text { Methanol Conversion } \\
\text { (reactant loss) }^{c}\end{array}$} & \multicolumn{2}{|c|}{$\begin{array}{c}\text { Mass Balance Closure } \\
\qquad(100 \%)\end{array}$} \\
\hline $240{ }^{\circ} \mathrm{C}$ & \multicolumn{2}{|l|}{$9.9 \mathrm{E}-02$} & \multicolumn{2}{|c|}{$1.1 \mathrm{E}-01$} & \multicolumn{2}{|r|}{93} \\
\hline $250{ }^{\circ} \mathrm{C}$ & \multicolumn{2}{|l|}{$1.9 \mathrm{E}-01$} & \multicolumn{2}{|c|}{$2.1 \mathrm{E}-01$} & \multicolumn{2}{|r|}{92} \\
\hline $260{ }^{\circ} \mathrm{C}$ & \multicolumn{2}{|l|}{$2.9 \mathrm{E}-01$} & \multicolumn{2}{|c|}{$3.1 \mathrm{E}-01$} & \multicolumn{2}{|r|}{94} \\
\hline $270{ }^{\circ} \mathrm{C}$ & \multicolumn{2}{|l|}{$3.9 \mathrm{E}-01$} & \multicolumn{2}{|c|}{$4.1 \mathrm{E}-01$} & \multicolumn{2}{|r|}{94} \\
\hline $280^{\circ} \mathrm{C}$ & \multicolumn{2}{|l|}{$5.3 \mathrm{E}-01$} & \multicolumn{2}{|c|}{$5.6 \mathrm{E}-01$} & \multicolumn{2}{|r|}{95} \\
\hline
\end{tabular}

Table S4: The amount of catalysts used for methanol carbonylation reactivity measurement with different Re weight loadings.

\begin{tabular}{|c|c|c|c|}
\hline & Catalyst weight (mg) & Balanced SBA-15 (mg) & $\mathbf{S i O}_{2}$ as dilution (mg) \\
\hline $1 \mathrm{wt} \% \mathrm{Re} / \mathrm{SiO}_{2}$ & 100 & 0 & 400 \\
\hline $5 \mathrm{wt.} \% \mathrm{Re} / \mathrm{SiO}_{2}$ & 20 & 80 & 400 \\
\hline $10 \mathrm{wt} \% \mathrm{Re} / \mathrm{SiO}_{2}$ & 10 & 90 & 400 \\
\hline $20 \mathrm{wt} \% \mathrm{Re} / \mathrm{SiO}_{2}$ & 5 & 95 & 400 \\
\hline $\mathrm{Re}_{2} \mathrm{O}_{7}$ mixed with $\mathrm{SiO}_{2}$ & 1 & 99 & 400 \\
\hline
\end{tabular}

Table S5: The amount of catalysts used to promote methanol conversion during methanol carbonylation reaction with 10 wt.\% $\mathrm{Re} / \mathrm{SiO}_{2}$.

\begin{tabular}{|c|c|c|}
\hline Catalysts weight (mg) & Balanced SBA-15 (mg) & $\mathbf{S i O}_{\mathbf{2}}$ as dilution (mg) \\
\hline 30 & 270 & 600 \\
\hline 60 & 240 & 600 \\
\hline 90 & 210 & 600 \\
\hline 180 & 120 & 600 \\
\hline 300 & 300 & 600 \\
\hline 600 & 0 & 600 \\
\hline
\end{tabular}


Table S6: Structural parameters for EXAFS fits of standard Re powder ( 2 shell fit), and the model with two Re-O scattering paths for the 5, 10, and $20 \mathrm{wt} . \%$ Re catalysts after oxidation.

\begin{tabular}{|c|c|c|c|c|c|c|c|}
\hline Sample & Path & $\mathrm{S}_{0}^{2}$ & $\mathbf{N}$ & $\Delta \mathrm{E}_{\mathbf{0}}$ & $\mathbf{R}$ & $\sigma^{2} \cdot 10^{3}$ & R-factor \\
\hline \multirow{2}{*}{ Re powder } & Re-Re & \multirow{2}{*}{$\begin{array}{c}0.73+/- \\
0.04\end{array}$} & 12 & \multirow{2}{*}{$7.2+/-0.5$} & $2.74+/-0.002$ & $3.5+/-0.2$ & \multirow{2}{*}{$0.56 \%$} \\
\hline & Re-Re & & 6 & & $3.87+/-0.008$ & $4.2+/-0.7$ & \\
\hline \multirow{2}{*}{$\begin{array}{l}5,10,20 \mathrm{wt} \% \\
\text { post-oxidation }\end{array}$} & $\mathrm{Re}-\mathrm{O}$ & \multirow{2}{*}{0.73} & 3 & \multirow{2}{*}{$5.9+/-1.4$} & $1.69+/-0.01$ & $1.0+/-0.5$ & \multirow{2}{*}{$0.79 \%$} \\
\hline & $\mathrm{Re}-\mathrm{O}$ & & 1 & & $1.77+/-0.04$ & $4.4+/-3.6$ & \\
\hline
\end{tabular}

Calculation of methanol conversion to each product for mass balances calculations

Conversion $_{\mathrm{AA}}=\frac{\mathbf{n}_{\mathrm{AA}}}{\mathbf{n}_{\mathrm{MeOH}}}$

Conversion $_{\mathrm{DME}}=\frac{2 \mathrm{n}_{\mathrm{DME}}}{\mathrm{n}_{\mathrm{MeOH}}}$

Conversion $_{\mathrm{MA}}=\frac{2 \mathrm{n}_{\mathrm{MA}}}{\mathrm{n}_{\mathrm{MeOH}}}$

Conversion $_{\text {Formaldehyde }}=\frac{\mathbf{n}_{\text {Formaldehyde }}}{n_{\mathrm{MeOH}}}$

$\mathrm{n}_{\mathrm{AA}}, \mathrm{n}_{\mathrm{DME}}, \mathrm{n}_{\mathrm{MA}}, \mathrm{n}_{\text {Formaldehyde }}$ : the concentration of acetic acid, dimethyl ether, methyl acetate and formaldehyde in the effluent.

$\mathrm{n}_{\mathrm{MeOH}}$ : the concentration of methanol entering the reactor.

Derivation of rate expression for acetic acid formation

1. $\mathrm{CH}_{3} \mathrm{OH}(\mathrm{g})+\stackrel{\mathrm{O}}{\mathrm{O}} \mathbb{\mathrm { O }}_{\mathrm{R}}^{\mathrm{I}}=\mathrm{O}$

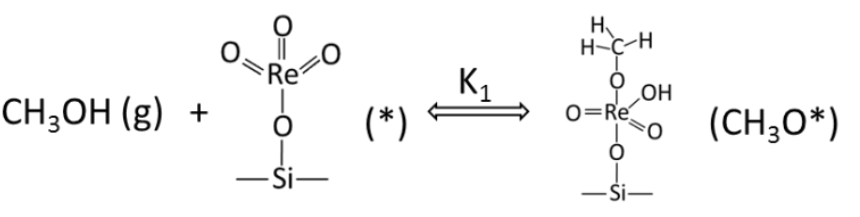

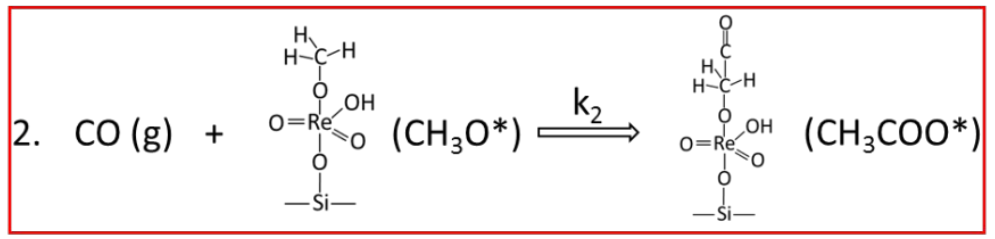

3.

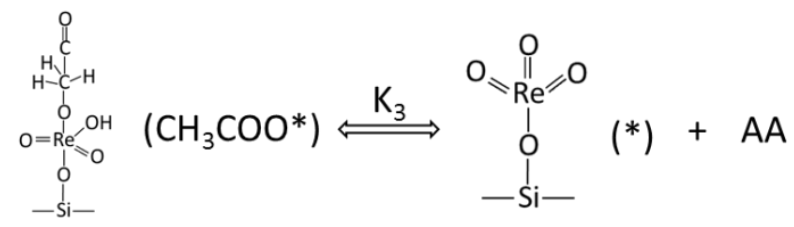


Acetic acid formation can be written down with 3 main elementary steps, in which CO insertion (step 2) is the rate limiting step and methoxy species are the most abundant surface intermedia (MASI). Step 1 and step 3 are assumed to be equilibrated. So, from step 1, the equilibrium constant can be written as:

$$
\mathrm{K}_{1}=\frac{\left[\mathrm{CH}_{3} \mathrm{O} *\right]}{\mathrm{P}_{\mathrm{CH} 3 \mathrm{OH}}[*]}
$$

After the rearrangement, the available site $\left[{ }^{*}\right]$ can be expressed as:

$$
[*]=\frac{\left[\mathrm{CH}_{3} \mathrm{O} *\right]}{\mathrm{K}_{1} \mathrm{P}_{\mathrm{CH}_{3} \mathrm{OH}}}
$$

With the assumption that the methoxy is the MASI, we have:

$$
\left[\mathrm{CH}_{3} \mathrm{O} *\right]+[*]=1
$$

Inserting equation (2) into (3), we can get:

$$
\left[\mathrm{CH}_{3} \mathrm{O} *\right]+\frac{\left[\mathrm{CH}_{3} \mathrm{O} *\right]}{\mathrm{K}_{1} \mathrm{P}_{\mathrm{CH}_{3} \mathrm{OH}}}=1
$$

Then, the expression for methoxy surface concentation can be written as:

$$
\left[\mathrm{CH}_{3} \mathrm{O} *\right]=\frac{\mathrm{K}_{1} \mathrm{P}_{\mathrm{CH}_{3} \mathrm{OH}}}{1+\mathrm{K}_{1} \mathrm{P}_{\mathrm{CH}_{3} \mathrm{OH}}}
$$

Acetic acid formation rate can be written as:

$$
\text { AA Production Rate }=\mathrm{k}_{2} \mathrm{P}_{\mathrm{CO}}\left[\mathrm{CH}_{3} \mathrm{O} *\right]
$$

Substituting (5) into this equation, we can get:

$$
\text { AA Production Rate }=\frac{\mathrm{k}_{2} \mathrm{~K}_{1} \mathrm{P}_{\mathrm{CO}} \mathrm{P}_{\mathrm{CH}_{3} \mathrm{OH}}}{1+\mathrm{K}_{1} \mathrm{P}_{\mathrm{CH}_{3} \mathrm{OH}}}
$$

\section{References}

(1) Tysoe, W. T.; Zaera, F.; Somorjai, G. A. An XPS Study of the Oxidation and Reduction of the Rhenium-Platinum System under Atmospheric Conditions. Surf. Sci. 1988, 200 (1), 1-14. (2) Falcone, D. D.; Hack, J. H.; Klyushin, A. Y.; Knop-Gericke, A.; Schlögl, R.; Davis, R. J. Evidence for the Bifunctional Nature of Pt-Re Catalysts for Selective Glycerol Hydrogenolysis. ACS Catal. 2015, 5 (10),

(3) Clarke, D.B.; Lee, D; Sandoval, M. J.; Bell, A. T. Infrared Studies of the Mechanism of Methanol Decomposition on $\mathrm{Cu} / \mathrm{SiO}_{2}$. J. Catal. 1994, 150, 81-93

(4) Liu, J.; Zhan, E.; Cai, W.; Li, J.; Shen, W. Methanol Selective Oxidation to Methyl Formate over $\mathrm{ReO}_{\mathrm{x}} / \mathrm{CeO}_{2}$ Catalysts. Catal. Letters 2008, 120 (3-4), 274-280. 